\title{
Un engagement envers l'action : SCPH 2015 et le Plan directeur pour la pharmacie
}

\author{
par Jason Howorko
}

Caisir l'occasion d'orienter l'avenir de notre profession ou être $\checkmark$ prêt à vivre avec le fait que nous en avions l'occasion, mais que nous n'avons rien fait.

C'est le message que j'ai retenu d'une allocution de la $\mathrm{D}^{\mathrm{re}}$ Linda Strand, l'une des créatrices du modèle des soins pharmaceutiques, lors de la Conférence nationale annuelle de l'Association des pharmaciens du Canada (APhC). De mon point de vue, c'était une allocution fantastique. En fait, cela m'a motivé personnellement. En revanche, j’étais déçu d'entendre les commentaires d'autres personnes dans le corridor après son allocution. Des commentaires comme "C'était des conneries" et « Ça n'arrivera jamais ». Eh bien, ces commentaires m'ont fait réaliser que notre profession traverse une crise : non pas une crise parce que notre profession évolue, mais un crise parce que de nombreux pharmaciens ignorent complètement les changements qui sont imminents ou qu'ils ne sont pas prêts à les saisir.

Heureusement, pour beaucoup d'entre nous qui pratiquons dans les établissements de santé, le changement n'est rien de nouveau. Au cours des années, les pharmaciens d'hôpitaux ont apprivoisé de nouvelles technologies, étendu leur champ de pratique et celui des techniciens en pharmacie, et développé des façons novatrices de prodiguer des soins à nos patients avec des ressources limitées. Aujourd'hui, les pharmaciens du Canada ont deux nouvelles occasions d'orienter l'évolution de leur profession.

En janvier 2008, la SCPH a lancé le projet SCPH 2015, un projet progressif, basé sur les résultats, communautaire, pour faire évoluer la pratique de la pharmacie hospitalière. Les données initiales ont été recueillies cette année et les services de pharmacie hospitalière ainsi que leurs pharmaciens à l'échelle du pays se sont mis à la tâche pour atteindre les objectifs du projet SCPH 2015.

Alors que la SCPH développait son projet SCPH 2015, les organismes de régulation de la pharmacie, les associations de pharmacie provinciales et nationales (dont la SCPH), les facultés de pharmacie, les étudiants en pharmacie ainsi que les pharmaciens et les techniciens en pharmacie des milieux hospitalier et communautaire créaient une vision commune de la profession, enchâssée dans le Plan directeur pour la pharmacie : La vision de la pharmacie (http://www.pharmacists.ca/ content/about_cpha/whats_happening/cpha_in_action/pdf/Pla nDirecteurPourLaPharmacie.pdf).

Alors pourquoi devrions-nous nous préoccuper de ces deux projets?

De nombreux pharmaciens d'hôpitaux ont la perception que le Plan directeur pour la pharmacie est destiné uniquement aux pharmaciens communautaires et d'autres estiment qu'il n'est aucunement pertinent pour eux parce qu'ils évoluent déjà dans un rôle de pratique clinique avancée. Toutefois, de nombreux pharmaciens sont aux prises avec des ressources matérielles et humaines limitées qui freinent l'avancement de leur pratique; or le Plan directeur pour la pharmacie est justement conçu pour composer avec ces problèmes. De plus, beaucoup d'objectifs du Plan directeur sont étroitement harmonisés avec ceux du projet SCPH 2015. Par conséquent, la SCPH demandera bientôt aux pharmaciens d'hôpitaux et aux services de pharmacie d'adhérer à ces deux projets en signant un "Engagement envers l'action ». En vous engageant ainsi officiellement, vous démontrerez que vous croyez à ce que vous faites en tant que professionnel et que vous croyez que tous les pharmaciens devraient exercer dans un cadre de pratique clinique évoluée.

La vision de l'évolution de la pratique est une réalité et c'est maintenant le moment d'y adhérer et de la faire vôtre. Cet automne, démontrez votre appui en signant l'Engagement envers l'action pour soutenir le projet SCPH 2015 et le Plan directeur pour la pharmacie. Cet engagement sera votre premier geste pour faire évoluer les choses et favoriser l'avancement de la profession.

[Traduction par l'éditeur]

Jason Howorko, B.S.P., B. SC., ACPR, est président et agent de liaison externe pour la $\mathrm{SCPH}$. 